\title{
BIBLIOGRAPHY OF DR. B.R. AMBEDKAR COLLECTION INTO VIVEKANAND LIBRARY, DR. BABASAHEB AMBEDKAR OPEN UNIVERSITY, AHMEDABAD.
}

\author{
Dr.Priyanki Vyas \\ Dr. Heena B. Ankuya
}

\begin{abstract}
The name of Dr. B.R. Ambedkar brings to our mind a Social worker, chief architect of the Constitution of India and a founding father of The Republic of India, the messiah of backward classes and women's rights. In this article, I have listed some famous books written by Dr. Ambedkar and also write a bibliography of collections of Vivekanand library, Dr. Babasaheb Ambedkar Open University, Ahmedabad. It is useful to find books and any materials of B.R.Ambedkar Sir. The main purpose of this article is with the use of this article, anyone can know the collection of B.R.Ambedkar Sir. More than 350 bibliographies I wrote in this article.
\end{abstract}

Keywords Babasaheb Ambedkar, Constitution.

"Caste is another name for control. Caste puts a limit on enjoyment. Caste does not allow a person to transgress caste limits in pursuit of his enjoyment. That is the meaning of such caste restrictions as interdining and inter-marriage...These being my views I am opposed to all those who are out to destroy the caste system." - Dr. B.R. Ambedkar, Annihilation of caste: The Annotated Critical Edition.

\section{Introduction}

Bhimrao Ramji Ambedkar, (born April 14, 1891, Mhow, India-died December 6, 1956, New Delhi), leader of the Dalits (Scheduled Castes; formerly called untouchables) and law minister of the government of India (1947-51). The life of Dr. Bhimrao Ambedkar was marked by struggles but he proved that every hurdle in life can be overcome with talent and firm determination. The hugest barrier in his life was the caste system according to which the family he was born in was considered 'untouchable'. 
Dr. Bhimrao Ambedkar was the chief architect of the Constitution of India and a founding father of the Republic of India. Dr. Ambedkar had knowledge of 9 languages like Hindi, Pali, Sanskrit, English, French, German, Marathi, Persian, and Gujarati. It is essential to tell that Babasaheb's personal library "Rajgrah" had more than 50,000 books and it was the world's largest private library. Dr. Babasaheb Ambedkar had mastery in 64 subjects.

The list of some famous books written by the Dr. Ambedkar Sir.

1. Castes in India: Their Mechanism, Genesis and Development-1916

2. Mook Nayak (weekly)-1920

3. The Problem of the Rupee: its origin and its solution-1923

4. Bahishkrut Bharat (India Ostracized)-1927

5. Janta (weekly) - 1930

6. The Annihilation of Caste-1936

7. Federation versus Freedom-1939

8. Thoughts on Pakistan- 1940

9. Ranade, Gandhi and Jinnah- 1943

10. Mr. Gandhi and Emancipation of Untouchables1943

11. What Congress and Gandhi have done to the Untouchables- 1945

12. Pakistan or Partition of India- 1945

13. State and Minorities- 1947

14. Who were the Shudras- 1948

15. Maharashtra as a Linguistic Province- 1948

16. The Untouchables- 1948

17. Buddha or Karl Marx- 1956

18. The Buddha and his Dhamma- 1957

19. Riddles in Hinduism- 2008

20. Manu and the Shudras

There are two most selling books of Ambedkar are the "Problem of the rupee" and "The Annihilation of caste". These two books are based on the economy of India and the social structure of India respectively. 
Towards Excellence: An Indexed, Refereed \& Peer Reviewed Journal of Higher Education/ Dr.Priyanki Vyas \&Dr. Heena Ankuya / Page 129-151

In this article, I have listed some popular books written by Dr. Ambedkar and also write a bibliography of collections of Vivekanand Library, Dr. Babasaheb Ambedkar Open University,Ahmedabad. It is helpful to find books and any matters of B.R.Ambedkar Sir. The main objective of this article is with the use of this article, anyone can know the collection of B.R.Ambedkar Sir. More than 400 bibliographies I addressed in this article. 


\section{Bibliography}

1 A.K., N. (2017). Dr. Ambedkar buddhism and social change . Delhi : Buddhist World Press .

2 Abraham, P. (2002). Ambedkar's contribution for economic planning and development-its relevance. New Delhi : Kanishka Publisher \& Distributors .

3 Abraham, P. (2002). Ambedkar's contribution for economic planning and development-its relevance. New Delhi : Kanishka Publisher \& Distributors .

4 ar, p. (2002). Ambdekar's contribution for economic planning and development its Ince. New delhi : Kanishka Publishing distrbutors .

5 Agrwal, K. (2013). Dr. ambedkar mahakavy part -2 . Delhi: B.R. Publishing .

6 Aharavala, K. L. (2013). Dr. ambedkar maha kavya. Delhi : B.R. Publishing Corporation .

7 Ahir, D. C. (1990). Legacy of Ambedkar (Bharat Ratna) . Delhi : B R Publishing corporation .

8 Ahir, D. C. (1990). Legacy of Ambedkar (Bharat Ratna). Delhi : B R Publishing corporation .

9 Ahir, D. C. (1990). The legacy of dr. amedkar (Bharat Ratna) . Delhi : B.R. Publishing Corporation .

10 Ahir, D. C. (1992). Ambedkar and Panjab. Delhi : B R Publishing corporation .

11 Ahir, D. C. (1996). Ambedkar buddhist revolution and counter-revolution in ancient India . Delhi : B R Publishing corporation .

12 Ahir, D. C. (1996). B R Ambedkar buddhist revolution and counter-revolution in ancient india . Delhi : B R Publishing corporation .

13 Ahir, D. C. (1997). Ambedkar and Indian constitution. Delhi : Low Price Publications .

14 Ahir, D. C. (1998). Ambedkar's vision of dhamma (an assessment). Delhi : B R Publishing corporation .

15 Ahir, D. C. (1998). Ambedkar`s Vision of Dhamma (an Assessment) . Delhi : B R Publishing corporation .

16 Ahir, D. C. (1998). Ambedkar`s Vision of Dhamma:an Assessment . Delhi : B R Publishing corporation .

17 Ahir, D. C. (2004). Buddhism and Ambedkar. Delhi : B R Publishing Corporation .

18 Ahir, D. C. (2007). Babasaheb Amedker Writings And Speeches . Delhi : B R Publishing corporation . 
19 Ahir, D. C. (2007). Dr babasaheb ambedkar writings and speeches : a ready reference manual (Vols. 1-17). Delhi : B.R. Publishing Corporation .

20 Ambedkar, B. (2017). Treasure of ambedkar series :- 1 : the evolutiion of provincial finance in british india : a Studay in the provincial decentralization of imperial finance. Delhi : Kalpaz Publications .

21 Ambedkar, B. (2017). Treasure of ambedkar series :- 2 : federation versus freedom . Delhi : Kalpaz Publications .

22 Ambedkar, B. (2017). Treasure of ambedkar series :- 3 pakistan or the partition of india . Delhi : Kalpaz Publications.

23 Ambedkar, B. (2017). Treasure of ambedkar series :- 4 What conggress and m.k.gandhi have done to the untouchables . Delhi : Kalpaz Publishing .

24 Ambedkar, B. (2017). Treasure of ambedkar series :- 5 who were the shudras ? : how they came to be the fourth verna in the indo-aryan society. Delhi : Kalpaz Publications .

25 Ambedkar, B. (2017). Treasure of ambedkar series :- 6 State and minorities : what are their rights and how to secure them in the constitution. Delhi : Kalpaz Publishing .

26 Ambedkar, B. (2017). Treasure of ambedkar series :- 8 the untouchables : who were they and why they became untouchables. Delhi : Kalpaz Publications .

27 Ambedkar, B. (2017). Treasure of ambedkar series :- 9 the buddha and his dhamma . Delhi : Kalpaz Publications .

28 Ambedkar, B. (2018). The problem of the rupee : its origin and its solution . Delhi : Kalpaz Publishing .

29 Ambedkar, B. R. (1994). Ashprushyo ane bhartiy samvidhan: B R Ambedkar. Ahmedabad : Navbharat sahitya mandir .

30 Ambedkar, B. R. (1997). Baba Saheb Ambedkar sampurna akshardeh Granth 10:asprushyo ane asprushyta- samajik, rajkiy, dharmik . Gandhinagar : Ambedkar Foundation.

31 Ambedkar, B. R. (1997). Baba Saheb Ambedkar sampurna akshardeh Granth 5: Aambedkar golmeji parisadma . Gandhinagar : Ambedkar Foundation .

32 Ambedkar, B. R. (1997). Baba Saheb Ambedkar sampurna akshardeh Granth 8:Hindu dharmna kut prashno. Gandhinagar : Ambedkar Foundation .

33 Ambedkar, B. R. (1998). Baba Saheb Ambedkar sampurna akshardeh Granth 12: Rupiyani samasya . Gandhinagar : Ambedkar Foundation .

34 Ambedkar, B. R. (1998). Baba Saheb Ambedkar sampurna akshardeh Granth 13:Shudro kon hta? Ane Bhartiy aaryona samajma teo chotha varnma kai rite ganaya? . Gandhinagar : Ambedkar Foundation . 
Towards Excellence: An Indexed, Refereed \& Peer Reviewed Journal of Higher Education/

Dr.Priyanki Vyas \&Dr. Heena Ankuya / Page 129-151

35 Ambedkar, B. R. (1998). Baba Saheb Ambedkar sampurna akshardeh Granth 14: Asprushyo kon hta? Ane teo asprushya kevi rite bnya? . Gandhinagar : Ambedkar Foundation .

36 Ambedkar, B. R. (1998). Baba Saheb Ambedkar sampurna akshardeh Granth 15: Pakistan ane Hindna bhagla. Gandhinagar : Ambedkar Foundation .

37 Ambedkar, B. R. (1998). Baba Saheb Ambedkar sampurna akshardeh Granth 16: Congress ane Gandhijie asprushyo mate shu kryu? . Gandhinagar : Ambedkar Foundation .

38 Ashok, P. (2016). Gabdhi and ambedkar \& indian dalits . Delhi : Wisdom Press .

39 Ashok, P. (2016). Gabdhi and ambedkar. Delhi : Wisdom Press .

40 B.R.Ambedkar. (2017). Treasure of ambedkar series :- 7 History of the indian currency and banking . Delhi: Kalpaz publications .

41 Bagchi, R. (1998). Mahatma Gandhi and B R Ambedkar on Islam and Indian Muslims . Delhi : B R Publishing corporation .

42 Bagehi, R. (2007). Mahatma Gandhi and Dr. b r Ambedkar. Delhi : B R Publishing corporation .

43 Bakshi, S. R. (1994). B R Ambedkar statesman and contitutionalist . New Delhi : Anmol Publication .

44 Bakshi, S. R. (2000). B R Ambedkar his political and social ideology vol-1. New Delhi: Deep \& Deep Publications Pvt Ltd .

45 Bakshi, S. R. (2000). B R Ambedkar his political and social ideology vol-2. New Delhi : Deep \& Deep Publications Pvt Ltd .

46 Bakshi, S. R. (2001). B R Ambedkar his political and social ideology . New Delhi : Deep \& Deep Publications Pvt Ltd .

47 BAOU. (n.d.). CALT - 01 Certificate In Dr Baba Saheb Ambedkar Life \& Thought. In Dr Ambedkarnu Jivan Charitra CALT-01 BLOCK 1. Ahmedabad: BAOU.

48 BAOU. (n.d.). CALT - 01 Certificate In Dr Baba Saheb Ambedkar Life \& Thought. In Dr Ambedkarnu Jivan Charitra CALT-01 BLOCK 3. Ahmedabad: BAOU.

49 BAOU. (n.d.). CALT - 01 Certificate In Dr Baba Saheb Ambedkar Life \& Thought . In Dr Ambedkarnu Jivan Charitra CALT-01 BLOCK 2. Ahmedabad: BAOU.

50 BAOU. (n.d.). CALT - 01 Certificate In Dr Baba Saheb Ambedkar Life \& Thought . In Dr Ambedkarnu Jivan Charitra CALT-01 BLOCK 4. Ahmedabad: BAOU.

51 BAOU. (n.d.). Dr.Babasaheb Ambedkar Opan Univarsity- FLCS. Ahmedabad: BAOU.

52 Bhandutia, B. J. (2007). B R Amedker. Jaipur: Oxford Book Company .

53 Bharathi, K. S. (1998). Encyclopaedia of Eminent thinkers: Political Thoughts of Ambedkar. New Delhi : Concept publishing company . 
54 Bhatnagar, R. (2006). Ambedkar Chintan Aur Vichar. Delhi : Jagatram And Sons .

55 Bhatnagar, R. (2006). Ambedkar Jeevan -Marm . New Delhi : Jagatram And Sans .

56 Bhatnagar, R. (2006). Ambedkar Jivan - Marm . New Delhi : Jagatram And Sans .

57 Bhatnagar, R. (2015). Yug Purush Ambedkar. Ahmedabad: Gurjar Granth prakashan .

58 Bhatnagar, R. M. (2005). Yugpurush Ambedkar. Delhi : Rajpal \& sons .

59 Bhatnagar, R. M. (2011). Dr. Ambedkar Jivan - Marma. New Delhi: Jagatram \& Sons

60 Bhatt, D. (1993). Ambedkar Nu Jivancharitra : dalit divakar Bhag 1 . Ahmedabad: Navbharat sahitya mandir .

61 Bhatt, D. (1993). Ambedkar Nu Jivancharitra : dalit divakar Bhag 2. Ahmedabad: Navbharat sahitya mandir .

62 Bhatt, D. (1993). Ambedkar Nu Jivancharitra : dalit divakar Bhag 3 . Ahmedabad: Navbharat sahitya mandir .

63 C.D., N. (2009). Ambedkar's perspective on buddhism and other religions . Delhi : Kalpaz Publishing .

64 C.M., G. (2011). Dr.B.R. Ambedkar and women's empowerment . Jaipur: ABD Publishing .

65 chahal, S. k. (2018). DR. B.R. Ambedkar The Maker of Modern India. Hariyana : Nirmal publishing house .

66 Chanchreek, K. L. (2006). B R Ambedkar. New Delhi : Shree Publishers .

67 Chandel, D. (2011). Manavadhikar Nehru aur Ambedkar. Jaipur : Pointer publishers.

68 Chandel, D. (2016). Manvadhikar me Ambedkar ka Yogdan . Jaipur: Rajsthan Hindi Granth Academy .

69 Chandra, R. (2000). Ambedkar science and society. New Delhi: Shree publishers .

70 Chandra, R. (2003). Ambedkar Era . New Delhi : Commonwealth publisher .

71 Chatterjee, D. (2004). Up Against Caste . Jaipur\&New Delhi : Rawat Publication .

72 Chauhan, G. (2006). Ambedkar Vichar Ane Vyavhar. Ahmedabad: Parshwa Publication .

73 Chauhan, S. S. (2004). Bharat me Dalit Chetna Gandhi Aur Ambedkar . Jaipur : RBSA publishers .

74 Chavan, S. (2001). Gandhi and Ambedkar savious of untouchables . Delhi : Bharatiya Vidya Bhavan .

75 Chavan, S. (2004). Congress, gandhi \& ambedkar assessment and observations of untouchability . Authorspress .

76 Chentharassery, T. H. (2000). Ambedkar on Indian history . Jaipur : Rawat 
Towards Excellence: An Indexed, Refereed \& Peer Reviewed Journal of Higher Education/ Dr.Priyanki Vyas \&Dr. Heena Ankuya / Page 129-151

publication .

77 Chitkara, M. G. (2002). Ambedkar and Social Justice. Neh Delhi : APH Publishing Corporation .

78 D.C, A. (2017). Dr. B.R. Ambedkar : buddhist revolution and counter revolution in ancient india . Delhi : Buddhist World Press .

79 D.M, b. (2015). Ha hun Dr. ambedkar balu chhu. Ahmedabad : Damini Publications .

80 D.R., J. (2011). Dynamics of ambedkar ideology . Jaipur: Sublime publecations .

81 datt, M. (2005). Gandhi, Ambedkar Aur Dalit . New Delhi : Radha Publication .

82 Desai, R. (1993). Ambedkar Granthmala . Ahmedabad : Kothari Prakashan Gruh .

83 Dhananajay, K. (2016). Vishvabhushan : Dr.Babasaheb ambedkar-manas Tatva ane vichar. Ahmedbad : Navsharjan Publication .

84 Dhani, S. L. (2007). B R Amedker Man Of Millennium for Social Justice . Delhi : Kalpaz publications .

85 Dhanraj, D. (2018). Dr. Ambedkar thought : Downtrodden and development. Delhi : B.R. Publishing Corporation .

86 Dharamveer. (2004). Ambedkar Ke Prashasnik Vichar. New Delhi: Vani prakashan .

87 Dharamveer. (2012). Matrasatta Pitrasatta Aur Jarsatta - Therigatha Ki Striyan Aur Ambedkar . New Delhi: Vani prakashan .

88 Dharmadhikari, N. (2003). Bhartiya Bandharannu Adhisthan . Ahmedabad: Navbharat sahitya mandir .

89 Dharmveer. (2004). Ambedkar ke Prashasnik Vichar. New Delhi : Vani prakashan .

90 Dharmveer. (2005). Thorigatha ki Striya Aur Ambedkar. New Delhi : Vani prakashan

91 Dhramveer. (2012). Therigatha ki striyan aur dr.ambedkar : matrasatta , pitrasatta aur jarsatta . New Delhi: Vani Prakashan .

92 Dhramveer. (2015). Dr.ambedkar ke prashasnik vichar. New Delhi: Vani Prakashan .

93 distributer, N. b. (2000). Biography of hisvision \& ideas. Ahmedabad : Niramal books distributer .

94 Dubey, A. K. (2002). Adhunikta ke aine me dalit. New Delhi : Vani Prakashan .

95 Dutt, P. K. (2014). Dr.ambedkar :asvikar ka sahas . New Delhi: Sasta Sahitya Mandal

96 Emanual, N. (2017). B.R. Ambedkar's philosophy in contemporary india . Delhi : Kalpaz Publications .

97 Gajrani, S. (2006). B R Ambedker. New Delhi : Commonwealth publisher .

98 Ganganiya, I. (2006). Ambedkarvadi Sahitya-Vimarash. New Delhi : Kitab Ghar .

99 Ganganiya, I. K. (2006). Ambedkarvadi Aalochana Ke Pratiman. New Delhi: Kitab 
Towards Excellence: An Indexed, Refereed \& Peer Reviewed Journal of Higher Education/ Dr.Priyanki Vyas \&Dr. Heena Ankuya / Page 129-151

Ghar .

100 Gatav, D. R. (2007). Ambedkar Aachr Vichar Sanhita . Jaipur : A B D publishers.

101 Gautam, Q. L. (2002). Babasaheb Ambedkar and Brahmanism . Delhi : B R Publishing corporation .

102 Gautama, Q. L. (2002). Baba Saheb Ambedkar and brahmanism . Delhi : B R Publishing corporation .

103 Gayakvad, G. K. (2009). Mahamanav BhimravRamji Ambedkar . Kanpur : Aman Prakashan .

104 Genva, K. (n.d.). Dr. Bhimrao Ambedkar ka Samajik Darshan . Ahmedabad: Khushbu Prakashak .

105 Ghatak, B. K. (1997). Ambedkar's Thought. New Delhi : A P H publishing corporation

106 Ghatak, B. K. (1997). Ambedkar`s Thought. New Delhi : A P H publishing corporation

107 Ghatak, B. K. (1997). Ambedkar's Thought. New Delhi : A P H publishing corporation

108 Ghohil, N. (2015). Mahamanav Dr. Ambedkar. Ahmedabad : Ranndede Prakshan .

109 Gohil, N. (2006). Bharatratna Bhimrav Ambedkar. Ahemdabad : Harsh Prakashan .

110 Gohil, N. (2015). Bharatratna Bhimrao Ambedkar. Amhedabad: Harsh Prakashan .

111 Gore, M. S. (1993). Social context of an ideology: Ambedkar's political and Social thoughts . New Delhi : Sage Publication .

112 Grover, V. (1992). B R Ambedkar. New Delhi : Deep \& Deep Publications Pvt Ltd .

113 Grover, V. (1998). Bhimrao Ramji Ambedkar. New Delhi : Deep \& Deep Publications Pvt Ltd .

114 Gupt, V. P. (1997). Bhimrao Ambedkar. New Delhi : Radha Publication .

115 Gupt, V. P. (2001). Bhimrav Ambedkar vyakti aur vichar. New Delhi : Radha Publication .

116 Gupt, V. P. (2001). Bhimrav Ambedkar vyakti aur vichar. New Delhi : Radha Publication .

117 Gupta, R. (1994). Ambedkar Aur Samajik Niyay . Delhi : Manak Publication Pvt Ltd .

118 Gupta, V. P. (1998). Maker of Modern India Series: Bhimrao Ramji Ambedkar. Delhi : Ambe Books .

119 Hardan, H. (2005). Bharatratna Bhimrav Ambedkar: Vyakti aur Vichar. Jaypur: Rastriy Sanskrut Sahitya Kendra.

120 Harsh, H. (2006). Bhimrao Ambedkar Jivan aur Darshan . Jaipur : Panchshil

Prakashan . 
121 Heggade, O. D. (1998). Economic thought of B R Ambedkar. New Delhi: Mohit publication.

122 Hemraj, M. (2013). Gabdhi and ambedkar :assessment and observation of untouchability . Jaipur: Book Enclave .

123 I.S., V. (2011). Indian freedom movement and Dr.b.r.ambedkar . Jaipur: ABD Publishing .

124 lyer, V. R. (1990). Ambedkar and the dalit future . Delhi : B R Publishing corporation .

125 Iyer, V. R. (1991). Ambedkar centenary : social justice and the undone vast. Delhi : B $\mathrm{R}$ Publishing corporation .

126 lyer, V. R. (1991). Ambedkar centenary : social justice and the undone vast. Delhi : B R Publishing corporation .

127 Jadhav, N. (1993). Ambedkar's Economic thought and Philosophy . Mumbai : Popular Prakashan .

128 Jadhav, N. (1993). Ambedkar`s Economic thought and Philosophy . Mumbai : Popular Prakashan .

129 Jadhav, P. (2007). Ambedkar's people in india . Jaipur : Book Enclave .

130 Janak, S. (2010). Dr. B.R. Ambedkar : the messiah of the downtrodden . Delhi : Kalpaz Publishing .

131 Jatava, D. R. (1993). Ambedkar and humanism: concept of humanism of Ambedkar . Jaipur : ABD Publishers .

132 Jatava, D. R. (1997). Critics of Ambedkar . Jaipur : Surabhi Publication .

133 Jatava, D. R. (1997). Critics of Ambedkar . Jaipur : Surabhi Publication .

134 Jatava, D. R. (1998). B R Ambedkar study in society and politics . Jaipur : National Publishing House .

135 Jatava, D. R. (1998). B R Ambedkar study in society and politics . Jaipur : National Publishing House .

136 Jatava, D. R. (2000). Politics of B R Ambedkar in National Movement . Jaipur \& Delhi : National Publishing House .

137 Jatava, D. R. (2001). Dynamics of Ambedkar Ideology . Jaipur : Sublime publication .

138 Jatava, D. R. (2001). Political philosophy of B R Ambedkar. Jaipur : National publishing house.

139 Jatava, D. R. (2001). Social thoughts of B R Ambedkar. Jaipur : ABD Publishers .

140 Jatava, D. R. (2001). Sociological Thoughts of B R Ambedkar . Jaipur : ABD Publishers .

141 Jatava, D. R. (2004). Ambedkar Ak Prakhar Vidrohi . Jaipur : ABD Publishers .

142 Jatava, D. R. (2004). Ambedkar The Prime mover. Jaipur: ABD Publishers. 
143 Jatava, D. R. (2004). A Farewell to Amedkar . Jaipur: ABD Publishers .

144 Jatava, D. R. (2004). B R Ambedkar a Vision of Man \& Morals . Jaipur : ABD Publishers .

145 Jatava, D. R. (n.d.). B R Ambedkar a vision of man \& morals . Jaipur : ABD Publisher

146 Jatva, D. R. (2001). Vishv dharm aur ambedkar . Jaipur : Sablaime Publication .

147 Jatva, D. R. (2005). Ambedkar Code of Couduct . Jaipur : ABD Publishers .

148 Jatva, D. R. (2005). Ambedkar Code of Couduct. Jaipur : ABD Publishers .

149 Jignashu, C. (2015). Jivan sangharsh . Ahmedabad: Navsarjan Publishing .

150 Jod, M. (2009). Dr. Bhimrao Ambedkar Ne Dharma Parivartan Kyon Kiya ? . Jodhpur: Minva Publication .

151 Jod, M. (n.d.). Samvidhan shilpi : Dr. Bheemaro Ambedkar. Ahmedabad: Khushbu Prakashak .

152 Jotikar, P. (2018). Ambedkari attarna pumada . gandhinagar: gujarat sahitya akadmi .

153 Joyprakash, A. (2014). ambdekar and dalit movement . New delhi : Omega Publications .

154 Jyotikar, P. (2016). Aarsh Drashta : Dr. babasaheb ambedkar . Ahmedabad: University granthnirman board .

155 Jyotikar, P. (2017). Dr. babsaheb ambedkar : Collection of histroical Poem (1930 A.D. To 1956 A.D.) . gandhinagar: gujarat sahitya akadmi .

156 Jyotikar, P. G. (1998). Aarshdrashta : Babasaheb Ambedkar. Ahmedabad : University granthnirman board .

157 Jyotikar, P. G. (1998). Aarshdrashta : Babasaheb Ambedkar. Ahmedabad : University granthnirman board .

158 Jyotikar, P. G. (2009). Ambedkar Vichardarshan. Ahmedabad : Babasaheb Ambedkar University .

159 K, S. R. (2010). Gandhi ambedkar and dalit . Jaipur: Aadi publications .

160 Kadam, K. N. (1993). Baba Saheb Ambedkar and the significance of his movement. Mumbai : Popular Prakashan .

161 Kadam, K. N. (1997). The Meaning of the Amedkarite Conversion to Buddhism and Other Essays . Mumbai : Popular Prakashan .

162 Kalpesh, P. (2016). Bharat-ratna Dr. Ambedkar. Ahmedabad: Paswa Publications .

163 kamal, K. L. (2006). Karamyogi Amedkar . Jaipur : RBSA publishers .

164 Kanagali, C. S. (1995). B R Ambedkar writer statesman. New Delhi : CBS Publisher \& Distributors .

165 Kanta, K. (2017). Dr b.r.ambedkar's vision of nation-building . Delhi: Kalpaz 
Towards Excellence: An Indexed, Refereed \& Peer Reviewed Journal of Higher Education/ Dr.Priyanki Vyas \&Dr. Heena Ankuya / Page 129-151

publications .

166 Keer, D. (2003). Vishvabhushan Babasaheb Ambedkar. Ahmedabad : Ami Publications .

167 keer, D. (2013). Dr. ambedkar : jivan ane karya. Ahmedabad : Navbhart sahitya madir .

168 Kesava Kumar, P. (2014). Political philosophy of ambedkar : an inquiry into the theoretical foundation of the dalit movement. Delhi : Kalpaz Publishing distrbutors .

169 Khan, N. H. (2001). B R Ambedkar on federalism, ethnicity and gender justice. New Delhi : Deep \& Deep Publications Pvt Ltd .

170 Khuman, M. (2016). Vilayatthi Babasaheb Aambedkare Lakhela Patro. Ahmedabad: Navsarjan Publication .

171 Khuman, M. V. (2013). Dr.ambedkar na bhasha no-1. Ahmedabad : Navsarjan Publishing .

172 Khuman, M. V. (2014). Dr.ambedkar na bhasha no-2. Ahmedabad : Navsarjan Publishing .

173 Khuman, M. V. (2014). Dr.ambedkar na bhasha no-3. Ahmedabad : Navsarjan Publishing .

174 Khuman, M. V. (2017). Bhartiy bandharan nu adhisthan. Ahmedabad: Navbhart sahitya madir .

175 Khuman, M. V. (2018). Dr.ambedkar na bhasha no-4. Ahmedabad : Navsarjan Publishing .

176 Kir, D. (1993). Ambedkar jeevan ane kary. Ahmedabad : Navbharat sahitya mandir .

177 Kir, D. (1996). Baba Saheb Ambedkar jeeva charit. New Delhi : Popular Prakashan.

178 Kir, D. (2007). Babasaheb Aambedkar. Mumbai : Popular Prakashan .

179 Krishan, A. (1997). Ambedkar and Gandhi emanclpators of untouchables in modern India . Mumbai : Himalaya Publishing House .

180 Kumar, R. (2006). Babasaheb Ambedkar Ki Vani . New Delhi : Gnan Publishing .

181 Kumar, R. (2007). Voice of Babasaheb Amedkar. New Delhi : Gyan publishing house

182 Kumar, R. (2008). Ambedkar and his writings : A look for new generation. Delhi : Kalpaz Publishing .

183 kumar, r. (2010). encyclopaedia of b r ambedkar. New Delhi : Commonwealth publisher .

184 Kumar, R. (2011). Ambedkar and Constitution . New Delhi: Commonwealth Publishers .

185 Kumar, R. (2011). Ambedkar and politics . New Delhi : Commonwealth Publishers . 
186 Kumar, R. (2011). Ambedkar and Religion . New Delhi : Commonwealth Publishers .

187 Kumar, R. (2011). Ambedkar and Society . New Delhi : Commonweal Publishers pvt.Itd.

188 Kumar, R. (2011). Ambedkar and the dalit. New Delhi : Commonwealth Publishers .

189 Kumar, R. (2011). Ambedkar and women . New Delhi: Commonweal Publishers pvt.Itd .

190 Kumar, R. (2011). Dr. B.R. Ambedkar : life and works . New Delhi : Commonweal Publishers pvt.Itd .

191 Kumar, R. (2011). Economic thought of B.R. Ambedkar. New delhi : Commonweal Publishers pvt.Itd .

192 Kumar, R. (2011). Perspectives on ambedkar thought. New delhi : Commonweal Publishers pvt.Itd .

193 Kumar, R. (2018). Encyclopaedia of dr. b.r. ambedkar (Vol. 1). New delhi: Commonweal Publishers pvt.Itd.

194 Kumar, R. (2018). Encyclopaedia of dr. b.r. ambedkar (Vol. 2). New delhi : Commonweal Publishers pvt.Itd .

195 Kumar, R. (2018). Encyclopaedia of dr. b.r. ambedkar (Vol. 4). New delhi: Commonweal Publishers pvt.Itd.

196 Kumar, R. (2018). Encyclopaedia of dr. b.r. ambedkar (Vol. 5). New delhi: Commonweal Publishers pvt.Itd.

197 Kumar, R. (2018). Encyclopaedia of dr. b.r. ambedkar (Vol. 6). New delhi: Commonweal Publishers pvt.Itd.

198 Kumar, R. (2018). Encyclopaedia of dr. b.r. ambedkar (Vol. 7). New delhi: Commonweal Publishers pvt.Itd.

199 Kumar, R. (2018). Encyclopaedia of dr. b.r. ambedkar (Vol. 8). New delhi: Commonweal Publishers pvt.Itd.

200 Kumar, R. (2018). Encyclopaedia of dr. b.r. ambedkar (Vol. 9). New delhi: Commonweal Publishers pvt.Itd.

201 Kumar, R. (2018). Encyclopaedia of dr. b.r. ambedkar (Vol. 10). New delhi: Commonweal Publishers pvt.Itd.

202 Kumar, R. (2018). Encyclopaedia of dr. b.r. ambedkar (Vol. 3). New delhi : Commonweal Publishers pvt.Itd.

203 Kumar, R. V. (2012). Bhimrav ambedkar. Patna: D. S. Books .

204 Kumar, S. S. (2010). Dr. B.R.Ambedkar : a study of indian society . Jaipur: ABD Publishing .

205 Kumar, S. S. (2010). Political and social thought of ambedkar . Jaipur: ABD 
Towards Excellence: An Indexed, Refereed \& Peer Reviewed Journal of Higher Education/ Dr.Priyanki Vyas \&Dr. Heena Ankuya / Page 129-151

Publishing .

206 Lal, S. (1998). Ambedkar and Nation-Building . Jaipur : Rawat publication .

207 Limaye, M. (2008). Manu Gandhi And Ambedkar And Other Essays . Delhi : Gian Publishing House .

208 Limbale Sharankumar. (n.d.). Pragyasurya : babsaheb Ambedkar. New Delhi: Vani Prakashan .

209 Limye, M. (2002). Babasaheb Ambedkar eak chintan. Delhi : Atmaram \& Sons .

210 Madhukar, T. (2018). Encyclopedia of Bhimrao Ambedkar (Vol. 1). Jaipur: ABD Publishers.

211 Madhukar, T. (2018). Encyclopedia of Bhimrao Ambedkar (Vol. 2). Jaipur: ABD Publishers.

212 Madhukar, T. (2018). Encyclopedia of Bhimrao Ambedkar (Vol. 3). Jaipur: ABD Publishers.

213 Madhukar, T. (2018). Encyclopedia of Bhimrao Ambedkar (Vol. 4). Jaipur: ABD Publishers.

214 Madhukar, T. (2018). Encyclopedia of Bhimrao Ambedkar (Vol. 5). Jaipur: ABD Publishers.

215 Madhukar, T. (2018). Encyclopedia of Bhimrao Ambedkar (Vol. 6). Jaipur: ABD Publishers.

216 Madhukar, T. (2018). Encyclopedia of Bhimrao Ambedkar (Vol. 7). Jaipur: ABD Publishers.

217 Madhukar, T. (2018). Encyclopedia of Bhimrao Ambedkar (Vol. 8). Jaipur: ABD Publishers.

218 Madhusoodan, T. (2013). Dr. Ambedkar ka shiksha darshan . New Delhi: Omega Publication .

219 Madhvi, T. (2011). Dr. Bhimrav ambedkar : jivan Sangharsh our vichar. Jaipur: Yking books .

220 Maheriya, C. (2015). Smatana senani : Dr. ambedkar. Ahmedabad : Sanskar Sahitya mandir .

221 Majumdar, A. K. (1997). Ambedkar and Social justice. New Delhi: The director publication division .

222 Makwana Ramesh H. (2011). Baba Saheb Ambedkar : Jivan Ane Kavan . Ahmedabad: Parshva publication .

223 Makwana Ramesh H. (2011). Bhimrao Ambedkar : Ek Samaj Sudharak. Ahmedabad: Parshva publication .

224 Makwana, K. (2016). Mahamanav: Dr. Babasaheb Ambedkar. Ahmedabad: Sahitya 
Towards Excellence: An Indexed, Refereed \& Peer Reviewed Journal of Higher Education/ Dr.Priyanki Vyas \&Dr. Heena Ankuya / Page 129-151

Sadhna Trust .

225 makwana, r. (2011). bhimrao ambedkar ek smaaj sudhark. Ahmedabad: Pashav Publication .

226 Makwana, R. H. (2013). Dr.babasaheb ambedkar jivan ane kavan . Ahmedabad: Paswa Publications .

227 Mal, P. (1999). Ashprushyata avm dalit chetna. Jaipur: Pointer Publisher.

228 Mal, P. (2008). Baba Saheb B R Ambedkar. Jaipur : Pointer publishers.

229 Mal, P. (2008). Babasaheb B R Ambedkar- Jivan aur Sangarsh . Jaipur : Pointer publishers .

230 Mal, P. (2009). Ambedkar aur Dalittidhhar Andolan. Jaipur : Avishkar publishers distributors .

231 Mal, P. (2011). Gandhi, Ambedkar avm Dalittidhar Andolan . Jaipur : Avishkar publishers distributors .

232 Mallaiah, L. C. (2006). The Relevance Of B R Amedker's Views On Indian Agricultural Development. Delhi : Abhijeet Publications .

233 Mandal, S. N. (1991). B R Ambedkar: His Thoughts and Observations . Jaipur \& Delhi: National publishing house .

234 Manohar, B. (2017). Dr. Bhimrao ambedkar. New delhi : Bhartiya Jnantith .

235 Mathew, T. (1991). Ambedkar: reform or revolution. New Delhi : Segment Books.

236 Mathur, P. (2010). Mahatma Gandhi aur Ambedkar. Jaipur : Ishika Publising House.

237 Meena, H. (n.d.). Bharat maie samajik nyay evam dalit sangharsh . Jaipur: Aadi Publication .

238 Mehra, S. (2002). Ambedkars` perspective on state caste and social justice. New Delhi : Sanjay Prakashan .

239 Mehta, C. (1991). Yug drasta Bhimrav Ambedkar. Jaipur : Malik \& Co .

240 Mehta, C. (2004). Bhimrav Ambedkar: Samvaidhanik Darshan . New Delhi : Aph Publishing Corporation .

241 Mehta, K. T. (2000). Durdarshi-Drashta : Babasaheb Ambedkar . rajpipla : K T Mehta

242 Meshram, L. G. (2008). Aur Babasaheb Ambedkar ne Kaha . Ahmedabad: Radhakrushna .

243 Michael, S. M. (1999). Dalits in modern India vision and values . New Delhi : Vistaar Publication .

244 Mina, K. (2011). Gandhi, Nehru, Ambedkar - Samajik Nyay avm Rajniti . Jaipur : Adi publications . 
Towards Excellence: An Indexed, Refereed \& Peer Reviewed Journal of Higher Education/ Dr.Priyanki Vyas \&Dr. Heena Ankuya / Page 129-151

245 Mina, K. (2011). Gandhi, Nehru, Ambedkar : Samajik Nyay Evam Rajniti . Jaipur: Aadi Publication .

246 Misra, K. (2011). B R Ambedkar As a human right leaders. Delhi: Navyug Books International .

247 Misra, K. (2011). B R Ambedkar As a human right leaders . Delhi: Navyug Books International .

248 Mohan, B. R. (2017). Dr.ambedkar : Chintan aur vichaar. Delhi: Jagatram and sans.

249 Mujawar, W. R. (2011). Social and political thought of B R Ambedkar (Vol. 1). Delhi: Manglam Publications.

250 Mujawar, W. R. (2011). Social and political thought of B R Ambedkar (Vol. 2). Delhi: Manglam Publications.

251 Murali Mohan, S. (2016). B R Ambedkar on Education (Vol. 1). Delhi: B R Publishing Corporation.

252 Murali Mohan, S. (2016). B R Ambedkar on Education (Vol. 2). Delhi: B R Publishing Corporation .

253 N, L. (2015). Ambedkar is Dying . Delhi : Ancient Publishing House .

254 Naik, C. D. (2003). Thoughts and Philosophy of B R Ambedkar. New Delhi : Sarup \& sons .

255 Naik, C. D. (2004). Ambedkar`s Perspective on Buddhism and other Religions . Delhi : Kalpaz publications .

256 Naik, C. D. (2006). Bauddh Vachan tatha Ambedkar Vichar. Delhi : Kalpaz publications .

257 Naik, C. D. (2007). Bhudhitv ke Agradut Dr. Ambedkar. Delhi: Kalpaj Publication .

258 Naik, C. D. (2015). Life and philosophy of Dr. babasaheb ambedkar (Vol. 1). Delhi: B.R. Publishing Corporation.

259 Naik, C. D. (2015). Life and philosophy of Dr. babasaheb ambedkar (Vol. 2). Delhi: B.R. Publishing Corporation.

260 Namishray, M. D. (2003). Caste and race : Comparative study of BR Ambedkar and Martin Luther King . Mumbai : Popular Prakashan .

261 Namishray, M. D. (2003). Caste and race : Comparative study of BR Ambedkar and Martin Luther King . Mumbai : Popular Prakashan .

262 Nanduram, N. (2008). Ambedker Dalits and Buddhism. New Delhi : Manak publication pvt ltd .

263 Narain, A. K. (1994). Ambedkar, Buddhism and Social Change . Delhi : B R Publishing corporation .

264 Narayan, D. (2010). Ambedkar gandhi and empowerment of dalit . Jaipur: ABD 
Towards Excellence: An Indexed, Refereed \& Peer Reviewed Journal of Higher Education/

Dr.Priyanki Vyas \&Dr. Heena Ankuya / Page 129-151

Publishing .

265 Narayan, D. (2016). Writings and speeches of ambedkar . Jaipur: Abd publishers .

266 Naval, C. (2010). Garibo ke masiha Bhimrav Ambedkar. jodhpur : Minarva Publication .

267 Naval, C. (2016). Garibo Ke Mashiha Dr. Bhimrao Ambedkar . Jodhpur: Arihant Prakashan .

268 Nitish, V. (2014). Rashtraneta : Dr. B.R. Ambedkar. Patna: Vani prakashan .

269 Omvedt, G. (1999). Dalits and the democratic revolution. New Delhi : Sage Publication .

270 P, J. (2009). Ambedkar Vichardarsan . ahmedabad: babasaheb ambedkar open university .

271 P.A., P. (2007). Yug pravartak patrakar Dr babasaheb ambedkar . maheshna: P.A.Parmar .

272 Paliwal, K. (2007). Ambedkar Samaj-Vyavastha Aur Dalit -Sahitya . New Delhi : Kitab Ghar .

273 Paliwal, K. (2007). Ambedkar Samaj-Vyavastha Aur Dalit -Sahitya . New Delhi : Kitab Ghar .

274 pandey, P. (2006). Ambedkar aur pan dindayal. Jaypur Bharat: ABD Publisher .

275 Pandyan, D. K. (n.d.). B R Ambedkar and the dynamics of neo-buddhism. New Delhi : Gyan Publishing House .

276 Parekh, H. (2002). Ambedkar pachchisi (Padyamay jivancharitra) . Nadiad : Harish Parekh .

277 Parmar Rameshchandra. (2001). Ambedkar Stabdi Gurjar Granthshreni . Ahmedabad: Gurjar Granthratna Karyalaya.

278 Parmar, M. K. ( 1997). Baba Saheb Ambedkar sampuran akshardeh Granth 7: prachin bharatma kraanti tatha pratikraanti, Budhha athva Karlmarcs . Gandhinagar : Ambedkar Foundation .

279 Parmar, M. K. (1997). Babaseheb Ambedkar Sampurna Akshardeh Granth 6: hindu dharm nu tatvagyan. Gandhinagar : Ambedkar Foundation .

280 Parmar, M. K. (1998). Baba Saheb Ambedkar sampuran akshardeh: Granth 3: Aambedkar Mumbai vidhan parishadma. Gandhinagar : Ambedkar Foundation .

281 Parmar, M. K. (1998). Baba Saheb Ambedkar sampurna akshardeh Granth 17:Shri Gandhi ane Asprushyoni mukti . Gandhinagar : Ambedkar Foundation .

282 Parmar, M. K. (1998). Baba Saheb Ambedkar sampurna akshardeh Granth 4: Aambedkar sayman comission sathe. Gandhinagar : Ambedkar Foundation .

283 Parmar, M. K. (1998). Baba Saheb Amedkar sampuran akshardeh Granth 11: East 
Towards Excellence: An Indexed, Refereed \& Peer Reviewed Journal of Higher Education/ Dr.Priyanki Vyas \&Dr. Heena Ankuya / Page 129-151

India company no vahivat ane nanakiy babato. Gandhinagar: Ambedkar Foundation

284 Parmar, P. (2000). Babasaheb Aambedkar . Ahmedabad : Gurjar Granthratna Karyalaya .

285 Parmar, P. A. (2004). Bharatiya Swatantrya Sangramma Aambedkarni Bhumika. Ahmedabad : Navbharat sahitya mandir .

286 Parmar, R. (1996). Ambedkar Shtabdi gurjar granth shreni : Kanundata Ambedkar . Ahmadabad : Gurajar Garanthratna Karyalaya .

287 Parmar, R. (1996). Ambedkar Shtabdi gurjar granth shreni : Vidhyapurush Ambedkar. Ahmadabad : Gurajar Garanthratna Karyalaya .

288 Parmar, R. (1996). Ambedkar Shtabdi gurjar granth shreni: Muknayak Ambedkar. Ahmadabad : Gurajar Garanthratna Karyalaya .

289 Parmar, R. (1996). Ambedkar Shtabdi gurjar granth shreni: Samajsudharak Ambedkar. Ahmadabad : Gurajar Garanthratna Karyalaya .

290 Parmar, R. (1996). Ambedkar Shtabdi gurjar granth shreni:Mahamanav Ambedkar . Ahmadabad : Gurajar Garanthratna Karyalaya .

291 Parmar, R. (2001). Aambedkar Shatabdi Gurjar Granthshreni . Ahmedabad : Gurjar Granthratna Karyalaya .

292 Parmar, T. (1999). Anusuchit jatiyo ke hito ke lie Ambedkar ki Bhumika. Jaipur : Publication Scheme .

293 Patel, G. (2015). Dr. Babashaheb Ambedkar Jivan Shandesh . Ahmedabad: Balvinod Prakashan .

294 Patel, G. N. (1998). Jo Babasaheb Ambedkar na hot to? . Ahmedabad : Ami Publication .

295 Patoriya, R. (2003). Ambedkar chritramay jivani . Delhi : Prabhat Prakashan .

296 Pradip, D. (2014). Ambedkar vision and philosophy . Jaipur: Abd publishers .

297 Prakash, P. (2002). Ambedkar, politics and scheduled castes . New Delhi : Ashish publishing house .

298 Prasad, S. N. (2010). Ambedkar and indian code of laws . Jaipur: ABD Publishing .

299 Prasad, S. R. (2014). Ambedkar and grievances of scheduled castes. Jaipur: Abd publishers .

300 Pratap, C. (2015). Dr. Ambedkar our dalit chetana . Delhi : Vishvabharti Publications .

301 Praveen, J. (2007). Ambedkar's people in india . New Delhi : Rajat Publications .

302 Preeti, N. (2015). Bhddha evem dhamma dem dr. ambedakara ka darsana . Delhi: Buddhist world press .

303 Premji, P. (2015). Dr. Babasheb ambedkar Jivan darshan . Ahmedabad : Gurjar 
Towards Excellence: An Indexed, Refereed \& Peer Reviewed Journal of Higher Education/ Dr.Priyanki Vyas \&Dr. Heena Ankuya / Page 129-151

grantha ratna karyalay .

304 R.K., A. (2010). B.R.Ambedkar \& his ideology . Jaipur: Sublime publecations .

305 R.K., K. (2015). Karl marx and babasaheb ambedkar : a comparative study . Delhi : Kalpaz Publications .

306 Rajan. (2011). Babasaheb Bhimrao Ambedkar. Ahmedabad : Divine Publications .

307 Rajasekhriah, A. M. (1989). B R Ambedkar the quest for social justice. New Delhi : Uppal Publishing House .

308 'rajvansh', G. D. (2011). The miraculous great man Dr. bhimrao ramji ambedkar. New Delhi : Commonwealth Publishers .

309 Rameshchandra, P. (2001). Muknaik Ambedkar. Ahmedabad : Gurjar grantha ratna karyalay .

310 Rameshchandra, P. (2015). Kanundata ambedkar. Ahmedabad : Gurjar Granth Ratan karalya .

311 Rameshchandra, P. (2015). Mahamanav ambedkar. Ahmedabad : Gurjar Granth Ratan karalya .

312 Rameshchandra, P. (2015). Samajsudharak ambedkar. Ahmedabad : gurjar shahitya bhavan .

313 Rana, H. (2016). Pratikar : A collection of ambedkar poems . Ahmedabad: Gujrati dalit shahitya academy .

314 Ranga, M. L. (2000). B R Ambedkar life, work and relevance. New Delhi : Manohar publishers and distributers .

315 Ransume, S. (1998). Babasaheb Aambedkar. Ahmedabad : Radhakrushna .

316 Rao, D. V. (2006). B R Anbedker. New Delhi : Manak publication pvt Itd.

317 Rao, J. S. (2002). B R Ambedkar his relevance today . New Delhi : Gyan Publishing House .

318 rattu, N. c. (2017). Dr. ambedkar : jeevan ke antim kuchh varsh. Delhi: kitabghar prakashan .

319 Ratu, N. (2006). Ambedkar Jeevan ke Antim Kuch Varsh. New Delhi : Kitab Ghar .

320 Roy, R. (2006). Gandhi \& Ambedkar. Delhi : Shipra publication .

321 S, J. D. (2011). Social philosophy of dr, B.R.Ambedkar . Jaipur: ABD Publishing .

322 S.K., K. (2012). B.R.Ambedkar \& caste mobilisation . Jaipur: Yking Books .

323 S.K., K. (2012). Gandhi nehru \& ambedkar jewels of india . Jaipur: Sublime publecations .

324 S.K., S. (2016). Dr.B.R. Ambedkar : budhism and dalit perspectives . New delhi :

Omega Publications . 
Towards Excellence: An Indexed, Refereed \& Peer Reviewed Journal of Higher Education/ Dr.Priyanki Vyas \&Dr. Heena Ankuya / Page 129-151

325 S.N., S. (2011). Dr. B.R.Ambedkar and emancipation of women . New delhi: Oxford Book Campany .

326 S.R., S. (2010). Life \& mission of B.R. Ambedkar. Jaipur: Sublime publications .

327 Sanjay, S. (2007). Dalit movement in india Role of dr. b.r. ambedkar. Jaipur: Book Enclave .

328 Sanjeev, M. (2010). Father of indian constitution : Dr.B.R. ambedkar. New delhi : Mahaveer \& Son's .

329 Sankrutyayan, R. (2009). Dr. Bhimrav ambedkar : jivan our vichar. New Delhi: Vani Prakashan .

330 Sanskrutyayan, R. (2002). Babasaheb Ambedkar. New Delhi : Vani prakashan .

331 Sashi, S. S. (1994). Baba Saheb Ambedkar sampuran akshardeh: Granth 1: Lakhano bhashano patro vagereno sangrah . New Delhi : Ambedkar Foundation .

332 Sashi, S. S. (1994). Baba Saheb Ambedkar sampuran akshardeh: Granth 2: Lakhano bhashano patro vagereno sangrah . New Delhi : Ambedkar Foundation .

333 SC/ST/Pune, C. O.-E. (2001). CD On Bharatratna Baba Saheb Ambedkar. Pune, Gujarat, India.

334 Senapathi, B. (2012). Dr. B.R. Ambedkar and untouchability. Delhi: Navyog books international.

335 Shabbir, M. (1997). B R Ambedkar study in law \& Society . Jaipur : Rawat publication

336 Shabbir, M. (2005). Ambedkar on Law, Constitution and Social Justice. Jaipur\&New Delhi : Rawat publication .

337 Shahare, M. L. (1993). Baba Saheb Ambedkar ki sangharshyatra avm sandesh. New Delhi : Sagment Books .

338 sharankumar, L. (2013). Pragyasurya . New Delhi: Vani Prakashan .

339 Sharma, K. (1992). Ambedkar and Indian Constitution . New Delhi : Ashish Publishing House.

340 Sharma, R. (2005). Ambedkar, Gandhi,Lohia Aur Bhartiy Etihas ki Samasya . New Delhi : Vani prakashan .

341 Sharma, R. (2005). Ambedkar ,Gandhi,Lohia Aur Bhartiy Etihas ki Samasya . New Delhi : Vani prakashan .

342 Sharma, R. (2005). Baba Saheb Ambedkar. Delhi : Rajpal \& sons .

343 Sharma, R. (2008). Gandhiji, Ambedkar,Lohiya aur Bhartiy Ithas ki Samsyae. New Delhi : Vani Prakashan.

344 Sharma, R. (2017). Gandhi, Ambedkar, Lohiya Aur Bhartiy Itihas Ki Samsyaye. New Delhi: Vani Prakashan . 
345 Sharma, S. (1992). Baba Saheb Ambedkar sangit suman. Ahmedabad: Aradhna art circle.

346 Sharma, S. P. (2003). B R Ambedkar A Crusader of Social Justice . Jaipur : RBSA publishers .

347 Sharma, S. R. (2006). Life and works of B R Ambedkar. Jaipur : Book Enclave .

348 Shivakeri, C. D. (2004). Ambedkar`s Political Philosophy . New Delhi : Anmol publications pvt Itd .

349 Shourie, A. (2005). Worshipping False Gods, Ambedkar \& the facts which have been erased . India: Noble Book Distributors.

350 Shourie, A. (2005). Worshipping False Gods, Ambedkar \& the facts which have been erased . India : Noble Book Distributors .

351 Singh, M. (2006). Bhimrao Ambedkar . llahabad : Lokbharti .

352 Singh, N. K. (2000). Ambadkar on religion. New Delhi : Anmol Publication.

353 Singh, N. K. (2000). Ambedkar on Religion. New Delhi : Anmol Publication .

354 Singh, N. K. (2000). Ambedkar on Religion. New Delhi : Anmol Publication .

355 Singh, R. (2008). Vikalp Ki Talash. Jaipur : National publishing house .

356 Singh, S. (1990). Ambedkar on Buddhist conversion and its impact. Delhi : Eastern Book Linkers .

357 Sinh, P. (2005). Ambedkar Aur Ak Manuvadi Vishleshan. Delhi : Kalpaz publications

358 Sinh, R. G. (2006). Ambedkar Samajik Nyay avm Parivartan . Jaipur \& Delhi: National Publishing House .

359 Sinh, S. (2010). Ambedkar, Gandhi aur Dalit patrakarita . New Delhi: Anamika Publishers and Distributers .

360 Siwach, J. R. (1999). Ambedkar on presidential and judicial impeachment . Delhi : Indian Publishers` Distributors .

361 Somaiya, B. (2007). Babasaheb Aambedkar. Gandhidam : Mishnari Prakashan .

362 Sosa, N. (2014). Rashtra nirmanna shilpi Dr. bhimrav ambedkar. Ahmedabad : Navsarjan Publishing .

363 Srivastava, S. (2007). Dalit Movement in India . Jaipur : Book Enclave .

364 Sukhdeorao, I. K. (2010). Dr babasaheb ambedkars : Approach to public finance and policy . Delhi : B.R. Publishing Corporation .

365 Thengri, D. (2004). Aambedkar And Samajik Kranti Ni Yatra . Ahmedabad : Samajik Samrasta Manch .

366 Thorat, S. (1998). Ambedkar role in economic planning and water policy . Delhi : Shipra Publication . 
367 Thorat, S. (2007). Amedker in Retrospect . Jaipur : Rawat Publications .

368 Tomar, J. P. (2010). Ambedkar Thought on Education. New Delhi : A P H Publishing Corporation .

369 Tripathi, M. (2011). Bhimrao Ambedkar -jivan, sangarsh,avm vichar . Jaipur : yking books .

370 Upadhyay, S. (2011). Ambedkar aur Dalit Chetana . Jaipur : Ishika Publishing House

371 Upadhyay, S. (2011). Ambedkar aur Dalit Chetana . Jaipur : Ishika Publishing House

372 Vakil, A. K. (1991). Gandhi Ambedkar dispute: an analytical study. New Delhi : Ashish publishing house.

373 Varma, R. P. (2011). Ambedkar aur unka Andolan. Delhi : Shree Natraj Prakashan .

374 Varma, R. P. (2011). Krantdarshi Yugpurush Ambedkar. Delhi : Kanti Publishar .

375 Verinder, G. (2003). Bhimrao Ramji Ambedkar. Ahmedabad: Honest Publication.

376 Verma, D. K. (2004). Ambedker Vision and Education of Weaker Sections. New Delhi : Manak publication pvt Itd.

377 Vidhyapith, B. S. (1999). Baba Saheb Ambedkar Marathavada Vidhyapith . Aurangabad: Ambedkar Foundation .

378 Vidyasagar, I. S. (2005). Concept of Humanism of Ambedkar . Jaipur : ABD Publishers .

379 Vimalkirti, M. (2008). Aur Babasaheb Ambedkar Ne Kaha. New Delhi : Radha Krishna Publication .

380 Virendrasing, Y. (2018). Dr. Bhimrav ambedkar our dalit. New Delhi: Omega Publication .

381 Virendrasing, Y. (2018). Dr. Bhimrav ambedkar our samajik parivrtan . New Delhi: Arpan Publications .

382 Virendrasing, Y. (2018). Dr. Bhimrav ambedkar our shiksha. New Delhi: Arpan Publications .

383 Virendrasinh, Y. (2018). Dr. bhimrao ambedkar our bhartiya samaj . New Delhi : Arpan Publications .

384 Virendrasinh, Y. (2018). Dr. Bhimrao ambedkar our bhartiya savidhan . New Delhi : Arpan Publications .

385 Virendrasinh, Y. (2018). Dr. Bhimrao ambedkar our Dharm darshan. New Delhi : Arpan Publications .

386 Vishvas, N. (2005). Rastraneta B R Ambedkar. Delhi : VidhyaNidhi .

387 Vivak, R. (1992). Ambedkar Jeevan Aur Adarsh . Jaipur: Malik \& Co . 
Towards Excellence: An Indexed, Refereed \& Peer Reviewed Journal of Higher Education/ Dr.Priyanki Vyas \&Dr. Heena Ankuya / Page 129-151

388 Vivekanand, T. (2011). Achoot matwad ke sach : gandhi aur ambedkar. New Delhi : Sasta sahitya mandal .

389 Yadav, V. (2011). Vishvi Sadi ke Mahanayak - Baba saheb Bhimrav Ambedkar . Delhi : Omega Publications .

390 Yadav, V. S. (2011). Bisavin Sadi ke Mahanayak Baba shaheb Dr. Bhimrao Ambedkar . Delhi: Omega Publication .

391 Yadav, V. S. (2011). Samtamulak Samaj avm Samajik Parivartan ke Yugpurush: Bhimrav Ambedkar. Delhi : Omega Publications .

392 Yadav, V. s. (2018). Dr.Bhimrav Ambedkar Aur Mahila. New Delhi : Arpan Publications .

393 Yagnik, H. P. (1998). Bhimrav Ambedkar : jansamanyna samuddharak. Ahmedabad : Ami Publication .

\section{Dr.Priyanki Vyas Associate Professor (Lib \& info. Sci.) Dr. Babasaheb Ambedkar Open University, Ahmedabad. \& Dr. Heena B. Ankuya}

